\title{
Processo de revisão da Política Nacional de Promoção da Saúde: múltiplos movimentos simultâneos
}

\author{
The review process of the National Health Promotion Policy: \\ simultaneous multiple movements
}

Dais Gonçalves Rocha ${ }^{1}$ Veruska Prado Alexandre ${ }^{2}$ Vânia Cristina Marcelo ${ }^{3}$ Regiane Rezende ${ }^{4}$ Júlia Devidé Nogueira ${ }^{5}$ Ronice Franco de Sá ${ }^{6}$
${ }^{1}$ Departamento de Saúde Coletiva, Universidade de Brasília. Campus Universitário Darcy Ribeiro, Asa Norte. 70910900 Brasília DF Brasil. daisrocha@yahoo.com.br ${ }^{2}$ Faculdade de Nutrição, Universidade Federal de Goiás.

${ }^{3}$ Faculdade de Odontologia, Universidade Federal de Goiás.

${ }^{4}$ Unidade Técnica de Determinantes Sociais da Saúde, Riscos para a Saúde, DCNT e Saúde Mental, OPAS/OMS Brasil.

${ }^{5}$ Faculdade de Educação Física, Universidade de Brasília.

${ }^{6}$ Núcleo de Saúde Pública e Desenvolvimento Social, Universidade Federal de

Pernambuco.
Abstract Public policy can be understood as the translation of government proposals and the aspirations of society. Driven by the advances and challenges of social transformation, the need for the coordination of agendas and limited social participation in the drafting of the 2006 National Health Promotion Policy (PNPS), the Ministry of Health and the Thematic Group on Health Promotion/Abrasco proposed the PNPS review. This article describes the steps in that process. It involves policy analysis conducted by literature review and document analysis from the "internal triangulation" standpoint. The revision process adopted multiple approaches on data gathering (Regional Workshops and Health Councils, Intra/Intersectorial Delphi and Electronic Questionnaire). It also used heterogeneous sources of information, different local contexts (five regions of Brazil) and peer validation. The results were systematically ordered with the aid of an analytical matrix organized by objectives, principles, guidelines, themes and actions, resulting in a draft of the new PNPS. Lastly, a national seminar was organized in which the results of the process and a synthesis of the revised text were presented, making it possible to identify process gains and the next steps for the actors involved with the new PNPS.

Key words Health promotion, Policies, Triangulation, Social participation
Resumo A política pública pode ser compreendida como a tradução de propósitos de governos e de anseios da sociedade. Impulsionados pelos avanços e desafios das transformações sociais, pela necessidade de articulação de agendas e a pequena participação social na elaboração da Política Nacional de Promoção da Saúde (PNPS), de 2006, o Ministério da Saúde e o Grupo Temático de Promoção da Saúde/Abrasco propuseram a revisão desta. Este artigo descreve os movimentos deste processo. Trata-se de uma análise de política, realizada por revisão de literatura e análise documental na perspectiva da "triangulação interna". O processo de revisão adotou: múltiplas abordagens na coleta de informações (Oficinas Regionais e com Conselhos de Saúde, Delphi Intra/Intersetorial e Questionário Eletrônico); fontes de informação heterogêneas; contextos locais diferentes (cinco regiões do Brasil) e validação por pares. Os resultados foram sistematizados com auxílio de uma matriz de análise organizada em torno de objetivos, princípios, diretrizes, temas e ações, resultando em uma minuta da nova PNPS. Por fim, foi realizado um seminário nacional para apresentar os resultados do processo e a síntese do texto revisado, possibilitando identificar ganhos do processo e próximos passos dos atores envolvidos com a nova PNPS.

Palavras-chave Promoção da saúde, Política, Triangulação, Participação social 


\section{Do processo de institucionalização da promoção da saúde no SUS à demanda de revisão da Política Nacional de Promoção da Saúde}

A aplicação dos princípios da promoção da saúde e a análise do processo de institucionalização desta no Sistema Único de Saúde (SUS), no Brasil, a partir da década de 1990, já foi objeto de diversos estudos ${ }^{1-6}$.

A despeito da porta de entrada da promoção da saúde no SUS ter sido a Estratégia de Saúde da Família ${ }^{7}$, com sua proposta de reorientação do modelo de atenção, não há questionamento de que o lançamento da Política Nacional de Promoção da Saúde (PNPS), pelo gestor federal do SUS, em $2006^{8}$, no contexto dos novos pactos de gestão, significou um marco para a definição de metas e a alocação de recursos específicos para a área no âmbito das esferas estadual e municipal $^{5,9}$. Ainda que haja evidências da escassez de recursos para as ações de promoção da saúde, tendo em vista que a previsão orçamentária inicial desta no Plano Nacional de Saúde, no período de 2008-2011, foi de somente $2,4 \%$ do total do orçamento ${ }^{10}$, e haja controvérsias quanto ao lócus da coordenação da PNPS 3,5 , a iniciativa de institucionalização de uma política pública desta natureza é reconhecida internacionalmente, juntamente com as experiências da Finlândia, na Europa $^{11}$ e do Chile, na América do Sul ${ }^{12}$.

Segundo Souza ${ }^{13}$, "não existe uma única, nem melhor, definição sobre o que seja política pública”. Para esta autora, a formulação de uma política pública deve ser compreendida como um processo por meio do qual "os governos traduzem seus propósitos em programas e ações, que produzirão resultados ou as mudanças desejadas no mundo real" ${ }^{\prime 3}$.

Loureiro e Miranda ${ }^{11}$, analisando o processo de formulação de políticas, ressaltam que estas resultam da interação e negociação entre organizações e atores-chave e dos compromissos que conseguem pactuar ao final. No caso brasileiro, a política de promoção da saúde aparece como uma proposta formal em um documento de $2002^{3,14}$, resultante de uma produção exclusivamente de técnicos e consultores do Ministério da Saúde. A aceitação destas "ideias" se ampliou e a PNPS, lançada em 2006, foi construída com a participação de representantes dos gestores do SUS (Conass e Conasems) e da academia. Esse processo ocorreu em duas etapas, sendo a primeira coordenada pela Secretaria Executiva do Ministério da Saúde durante a gestão de 2003-
2004, e a segunda, entre 2005 e 2006, já a partir da Secretaria de Vigilância em Saúde. Estes espaços e seus atores foram os únicos envolvidos, ainda que os autores que lideraram o processo de escrita em 2003-2004 reconhecessem a necessidade de um "diálogo constante com os movimentos sociais, conseguindo estabelecer formas de articulação mais permanentes e sustentáveis na medida em que nessa relação pode-se multiplicar possibilidades de enfrentamento dos problemas de saúde pública e qualificar cada vez mais as ações no Sistema Único de Saúde"1.

Pinder ${ }^{15}$, refletindo sobre o papel do gestor federal na implementação das ações de promoção da saúde no Canadá, afirma que "a introdução daquelas novas ideias no governo não foi 'muito difícil' e também foi possível 'ganhar a aceitação' destas ideias", no entanto, as realizações não seguiram a mesma abrangência e não foram tão efetivas quanto aquelas.

Faz-se necessário reconhecer que, a despeito da importância dos governantes liderarem este processo de formulação de uma política, "a essência da política pública se dá no contexto de embate em torno de ideias e interesses" ${ }^{\prime 13}$. Com esta perspectiva, um único segmento ou ponto de vista não é suficiente para atender às necessidades, para tal deve-se garantir transparência e participação democrática.

Em relação aos atores da sociedade civil, verificou-se que, no processo de formulação da PNPS de 2006, somente as instituições de ensino e pesquisa e o Grupo Temático de Promoção da Saúde e Desenvolvimento Sustentável da Associação Brasileira de Saúde Coletiva (GT PS) da Abrasco estiveram presentes.

O GT PS da Abrasco foi criado em 2002, a partir de uma Oficina do Pró-GT Promoção durante a Conferência Latino Americana de Educação e Promoção da Saúde, realizada em São Paulo-Brasil ${ }^{16}$. Este tem por missão: "Articular, congregar, mobilizar e promover a incorporação dos princípios, pressupostos da Promoção da Saúde, na produção de conhecimento, nas práticas, nas políticas públicas e nos modos de fazer saúde no Brasil, além de disseminar e trocar experiências e conhecimentos nos níveis nacional e internacional"17. Seus representantes foram convidados pontualmente, principalmente, nos anos de 2005 e 2006, para discutir a operacionalização da agenda e os editais da PNPS. O GT só se inseriu formalmente na composição do Comitê Gestor da PNPS, a partir de 2010, quando passou a atuar em cooperação mútua em diferentes momentos, com destaque para comissão de seleção e aprova- 
ção de editais para garantia de recursos da área e na organização de cursos e seminários. Cabe esclarecer que o Comitê Gestor da PNPS é um colegiado formado por representantes do Ministério da Saúde e órgãos a ele vinculados (Anvisa, Fiocruz), representantes do CONASS e Conasems, OPAS e sociedade civil (Abrasco).

Uma das principais defesas/"advocacy" dos representantes do GT tem sido a proposição da democratização e ampliação da participação na construção e implementação da PNPS, buscando assim a sua tradução em uma política pública sustentável. Para tal, tem assumido a postura de que é preciso que as ideias sejam construídas e difundidas de forma mais abrangente possível e considerando os diferentes segmentos populacionais e contextos locorregionais ${ }^{18,19}$.

Assegurar e favorecer a promoção da saúde da população é uma tarefa a ser compartilhada. A despeito dos diversos mecanismos de institucionalização da participação no SUS, mediante os conselhos, conferências, comitês gestores e intergestores bipartite e tripartite, entre outros, existem diversas barreiras para efetivar a articulação de governo, trabalhadores, usuários e/ou representantes da sociedade ${ }^{20-22}$.

Além da identificação dos atores e do reconhecimento de articulações e negociações, uma dimensão importante de análise de processos de formulação de políticas públicas é o reconhecimento de que esta é construída dentro de um contexto histórico e social ${ }^{23}$. Os estudos de Rocha $^{4}$ e Cruz $^{5}$ evidenciaram que a OPAS exerceu um papel estratégico durante os 08 anos de discussão sobre promoção da saúde no Ministério da Saúde brasileiro, até a sua institucionalização em uma política nacional. As agendas externas ou internacionais de "Cidades/Municípios Saudáveis", Estratégia Global da Organização Mundial de Saúde e as demandas sistematizadas pela Comissão Nacional de Determinantes Sociais da Saúde ${ }^{24}$ configuraram um cenário e induziram uma agenda que promoveu um debate permanente entre as visões de promoção da saúde comportamental e a promoção da saúde voltada para enfrentamento da determinação social do processo saúde-doença.

Em 2013, 7 anos após a implementação da PNPS, o Comitê Gestor da PNPS do Ministério da Saúde propôs uma ação de atualização e reflexão sobre esta, frente a compromissos assumidos pelo Governo brasileiro nos últimos anos relativos a novas demandas, políticas e agendas internacionais e nacionais decorrentes de eventos e momentos estratégicos ${ }^{25}$. Neste sentido, desta- cam-se como marcos do cenário deste processo de revisão:

- Plano Nacional Ações Estratégicas para Enfrentamento de Doenças Crônicas Não Transmissíveis - Ministério da Saúde ${ }^{26}$;

- Decreto $n^{\circ} 7.508$, de 28 de junho de $2011^{27}$. Regulamentação da Lei no 8.080/1990, na perspectiva da articulação interfederativa e regionalização da saúde;

- Conferência de Alto Nível da Organização das Nações Unidas - Doenças Crônicas não Transmissíveis ${ }^{28}$;

- Declaração da Conferência Mundial dos Determinantes Sociais da Saúde ${ }^{29}$;

- Declaração da Conferência Rio + 20 $0^{30}$;

- Declaração da $8^{a}$ Conferência Mundial de Promoção da Saúde, Saúde em todas as Políti$\mathrm{Cas}^{31}$;

Os debates e documentos oriundos destas agendas, assim como sua necessária articulação, impulsionaram a revisão da PNPS; considerando os avanços e desafios das transformações sociais. Ainda, a própria agenda da PNPS estava com seu prazo expirado, pois na sua reedição em 2010, as ações específicas priorizadas estavam ainda datadas para o biênio 2006-200732.

Considerando também os avanços desde a perspectiva intersetorial, mencionada como estratégia central nas referidas agendas, o processo de revisão da PNPS não poderia prescindir de envolver diferentes setores, como forma de ampliar o escopo desta política para além do setor saúde, visando o diálogo, a sinergia e a construção de possibilidades para fazer frente às iniquidades $\mathrm{e}$ aos determinantes sociais.

Para Serafim e Dias ${ }^{33}$ a análise de políticas públicas requer uma profunda compreensão de contextos que possibilitam o entendimento do seu processo de elaboração em uma perspectiva mutimétodos e inter/multidisciplinar.

A interação dos múltiplos atores "implicados" na construção de uma política pública não se dá de maneira automática e requer institucionalização de arranjos e mecanismos deliberadamente fomentados para fortalecimento da participação social. Ainda, reconhecendo que o Brasil, historicamente, tem um modelo de desenvolvimento excludente, o qual promove a concentração dos recursos políticos, econômicos e sociais e que, consequentemente, aumenta a desintegração social e as iniquidades em saúde, priorizou-se desencadear e/ou mobilizar múltiplos processos nas cinco macrorregiões do Brasil.

Considerando este contexto, este artigo objetiva descrever os múltiplos processos empreendi- 
dos na revisão da PNPS, no período de abril de 2013 a junho de 2014.

\section{Metodologia}

Fundamentado no modelo de análise de política pública proposto por Araújo Júnior e Maciel Filho $^{23}$, com enfoque nos atores e processo, realizou-se um relato de caso, construído a partir de revisão de literatura e da análise documental dos seguintes registros e fontes de informação: relatórios e memórias das sessões do Comitê Gestor da PNPS no período de 2013 a 2014; relatório das Oficinas Regionais de revisão da PNPS e encontros de sistematização de dados; apresentações em powerpoint dos diferentes atores e momentos da revisão realizadas no período; e-mails e webconferencias (Elluminate OPAS); e notas pessoais das autoras envolvidas neste artigo.

Além da leitura dos e-mails trocados durante este período, as autoras participantes da análise deste processo de revisão da PNPS solicitaram o envio das apresentações e relatórios da coordenadora de cada etapa ou "movimento de escuta" visando a localização e organização deste material documental.

Para o processo de meta-leitura dos dados coletados, manteve-se a perspectiva da "triangulação interna à própria abordagem". Segundo Minayo ${ }^{34}$, esta "consiste em olhar o objeto sob seus diversos ângulos, comparar os resultados de duas ou mais técnicas de coleta de dados e de duas ou mais fontes de informação". Esta foi, originalmente, adotada pelo grupo coordenador do processo de revisão da PNPS, o qual agregou os resultados dos múltiplos movimentos simultâneos à análise das agendas e compromissos do Governo brasileiro no cenário nacional e internacional.

\section{Resultados}

O processo de revisão da PNPS foi realizado por meio de uma parceria entre membros do GT Promoção da Saúde da Abrasco, do Comitê Gestor da PNPS e do Ministério da Saúde destacando-se a equipe do Departamento de Vigilância de Doenças e Agravos Não Transmissíveis e Promoção da Saúde (DEVDANTPS) e a Opas.

Múltiplos movimentos simultâneos foram utilizados como estratégia de escuta, mobilização e produção de sínteses e permitiram a elaboração coletiva da minuta da PNPS, revisada ao final do processo, sendo estes: oficinas com diferentes atores, tendo como prioridade as macrorregiões do país e membros do Conselho Nacional de Saúde, utilização do método Delphi com diferentes públicos (intrassetorial, intersetorial e universidades), disponibilização de questionário virtual via Form-SUS, oficinas de sistematização (realizada entre pares) e Seminário Nacional (Figura 1).

Tais movimentos não se constituíram em um processo de avaliação de uma política e sim em um processo de revisão amplamente debatido no âmbito do Comitê Gestor da PNPS, o qual definiu que o mesmo ocorreria a partir de oficinas regionais onde seria possível " [...] captar a diversidade do país nas suas cinco regiões e olhar para quem está, na prática, operacionalizando essa política, para saber o que ela ainda não contempla e o que está dando certo e precisa ser continuado. Buscar todas essas vozes para construir uma política que dialogue não só para dentro, mas também para fora do setor da saúde" ${ }^{\text {"34 }}$.

Participaram destes processos membros de diferentes segmentos: gestores, trabalhadores (da saúde e de outros setores), conselheiros de saúde (diferentes instâncias federativas), pesquisadores e professores vinculados a universidades, representantes de movimentos sociais e de usuários. A Tabela 1 consolida o número de participantes, segundo segmento autorreferido e estratégia de escuta ou mobilização para a revisão.

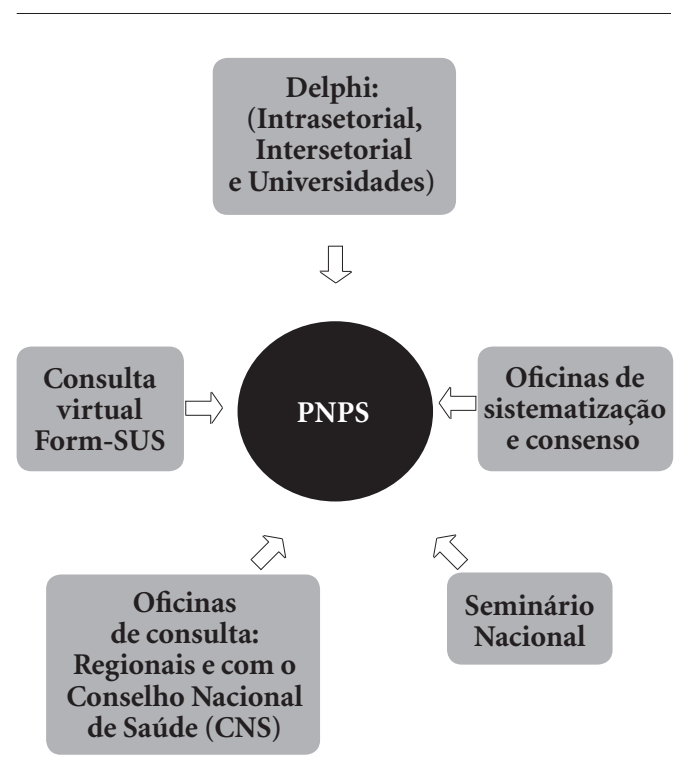

Figura 1. Múltiplos Movimentos Simultâneos utilizados para a revisão da Política Nacional de Promoção da Saúde - Brasil, 2014. 
Tabela 1. Número de participantes segundo segmento e estratégia de escuta ou mobilização para a revisão da Política Nacional de Promoção da Saúde, 2014.

\begin{tabular}{|c|c|c|}
\hline Estratégia de escuta e mobilização & No de participantes & Segmentos envolvidos \\
\hline FomSUS & 1.545 & $\begin{array}{l}\text { Secretaria Municipal de Saúde (883), } \\
\text { Trabalhador de Saúde (200), Ministério da } \\
\text { Saúde (126), Secretaria Estadual de Saúde (102), } \\
\text { Universidade (73), Usuário (41), Conselhos } \\
\text { de Secretários (26), Movimento Social (20), } \\
\text { Regional de Saúde (12), Comissão Intergestores } \\
\text { Regional (5), outros (57). }\end{array}$ \\
\hline DELPHI Intersetorial & 32 & $\begin{array}{l}\text { Secretaria Geral da Presidência da República, } \\
\text { Ministérios das Cidades e da Educação, } \\
\text { Secretarias de Estado, Prefeituras, Instituto de } \\
\text { Planejamento Urbano, ONGs, Fundação para o } \\
\text { Desenvolvimento Sustentável, Serviço Social da } \\
\text { Indústria, outros. }\end{array}$ \\
\hline DELPHI Intrassetorial & 39 & $\begin{array}{l}\text { Representantes de setores do Ministério da } \\
\text { Saúde relacionados à Promoção da Saúde. }\end{array}$ \\
\hline DELPHI universidades & 59 & $\begin{array}{l}\text { Coordenadores de grupos de pesquisa que } \\
\text { tinham a promoção da saúde como objeto } \\
\text { principal de estudo no Diretório de Grupos de } \\
\text { Pesquisa do CNPq. }\end{array}$ \\
\hline $\begin{array}{l}\text { Oficinas Regionais* e com o Conselho } \\
\text { Nacional de Saúde }\end{array}$ & 246 & $\begin{array}{l}\text { Representantes da: Gestão Municipal - gestores e } \\
\text { trabalhadores de diferentes setores (62), Gestão } \\
\text { Estadual - diferentes setores (35), Legislativo } \\
\text { [Vereadora (1), Presidência da Câmara } \\
\text { Municipal, Defensoria Pública (3), ONG e } \\
\text { Movimentos Sociais (13), Universidades (79), } \\
\text { Gestão Federal (MS), IBGE, Serviço Geológico } \\
\text { do Brasil (22), Conselhos profissionais (3). } \\
\text { Outros (7)] } \\
\text { Oficina Conselho de Saúde: Conselheiros } \\
\text { Estaduais (17), Conselheiros Municipais (4) } \\
\text { Conselho Nacional de Saúde (1). }\end{array}$ \\
\hline
\end{tabular}

A partir da análise dos resultados dos diferentes movimentos simultâneos foi possível realizar a triangulação considerando as seguintes perspectivas: 1) Múltiplas abordagens adotadas como métodos de coleta de informações ou estratégias de escuta para a revisão da PNPS (Oficinas Regionais e do Conselho Nacional de Saúde, Delphi Intrassetorial e Intersetorial, Questionário Eletrônico-FormSUS); 2) a heterogeneidade de atores como fontes das informações (profissionais de saúde, pessoas interessadas, trabalhadores em promoção, profissionais em outros setores, pesquisadores, professores, representantes institucionais, etc.). 3) Contextos locais considerando as diferentes regiões geográficas do Brasil.
Os Encontros de Sistematização possibilitaram a construção de uma matriz onde foi possível compor/modelar a triangulação dos resultados dos diferentes movimentos e construir coletivamente a minuta da PNPS que, posteriormente recebeu contribuições dos participantes do Seminário Nacional.

Passa-se a seguir à descrição das diferentes estratégias de escuta e mobilização utilizadas no processo de revisão da PNPS brasileira.

A estratégia das Oficinas foi desenvolvida adotando uma metodologia que seguiu o princípio da participação e regionalização, sendo planejados dois dias de atividades, com a participação máxima de 40 pessoas, a partir de debate 
sobre questões geradoras, trabalhos em grupos e técnicas projetivas que favorecessem resgatar as experiências, visões e sugestões para a revisão da $\mathrm{PNPS}^{34}$.

Os representantes do GT de Promoção da Saúde da Abrasco assumiram a mobilização em cada macrorregião, em consonância com os novos instrumentos de gestão do SUS, que delineiam uma nova governança regional. Destacase, entre estes, o Decreto no 7.508 , de 28 de junho de 2011, que dispõe sobre a articulação interfederativa com ênfase na equidade entre as regiões de saúde e estabelece o Contrato Organizativo de Ação Pública $(\mathrm{COAP})^{27}$. Para tal deve-se estimular a construção de consensos políticos (diretrizes, objetivos, regiões, resolutividade, acesso, regulação, mapa e responsabilidades).

A metodologia das Oficinas foi construída a partir de diferentes referências que possibilitaram ampliar os componentes da PNPS, construir coerência entres estes componentes, democratizar as decisões quanto às prioridades que deveriam ser explicitadas e construir redes de compromisso com a implementação da política. O "Marco de Referência do Guia de Avaliação Participativa de Municípios e Comunidades Saudáveis: recursos práticos para a ação"35,36 foi adaptado e utilizado como Marco de Referência para as oficinas, possibilitando a inserção dos valores como novo componente da PNPS, bem como a construção de coerência entre estes valores e os temas prioritários, as diretrizes, os eixos operativos e responsabilidade pela efetivação de estratégias e ações implicadas com a transformação da realidade a partir dos condicionantes e determinantes (Figura 2).

Como forma de democratizar as decisões sobre os temas a serem priorizados em cada região, foi utilizado o procedimento "Taking Stock", sugerido por Fetterman ${ }^{37}$, na metodologia "Empowerment Evaluation". Trata-se de uma maneira de priorizar eventos, fatos ou resultados de maneira democrática. Com este procedimento, cada um dos participantes (gestores, equipe técnica dos serviços, universidade e lideranças) pontuou os resultados com mesmo poder de escolha sobre a importância dos temas e eixos identificados. Também foi realizada uma discussão tendo como base a Lei Complementar no 141 , de janeiro de 2012, que define gastos em saúde, para fomentar a discussão sobre o papel do setor saúde, tanto no desenvolvimento de ações especificas como na mobilização de outros setores e na avaliação do impacto de outras políticas na saúde. Tal discussão contribuiu para a definição

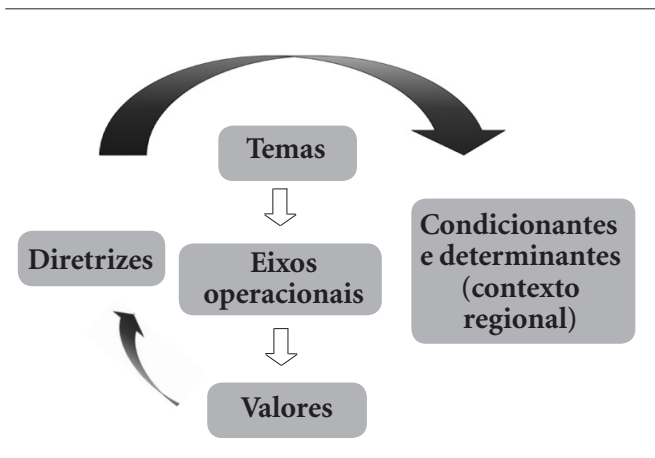

Figura 2. Marco de Referência utilizado durante as oficinas de revisão da Política Nacional de Promoção da Saúde, Brasil 2014*.

*Adaptado do Marco de Referência do Guia de Avaliação Participativa de Municípios e Comunidades Saudáveis: recursos práticos para a ação, OPAS/OMS, $2006^{35,36}$.

das responsabilidades do setor saúde para com a PNPS nas diferentes esferas de gestão.

Ao final de cada oficina, buscou-se constituir redes de compromisso regionais e locais, sensibilizando os participantes para a importância de uma mobilização permanente, de natureza pessoal e institucional, visando o fortalecimento da promoção da saúde. Foi proposta uma técnica projetiva com narrativa escrita, utilizando cartões postais das regiões onde foram realizadas cada oficina. Esta técnica foi anteriormente aplicada pela equipe da Associação Canadense de Saúde Pública da British Columbia, no Canadá, na Oficina que realizaram para uma discussão sobre "o Futuro da Promoção da Saúde" naquele país ${ }^{38}$. Os cartões postais foram organizados em um painel final que retratou as diferentes oficinas e suas características regionais, o qual foi exposto durante o Seminário Nacional.

Quanto ao total de oficinas, além de uma oficina teste, foram realizadas oficinas tendo como referências as 5 grandes regiões geográficas brasileiras (norte, nordeste, centro-oeste, sudeste e sul), e uma específica para Conselheiros nacionais e estaduais, que desenvolveram um exercício reflexivo de balanço da PNPS, para problematizar e pactuar valores, princípios, diretrizes e temas prioritários. Tendo ainda como pressuposto a participação, optou-se pela realização de uma oficina complementar da região sudeste em decorrência da pequena abrangência de representantes dos estados que compõem a região na primeira data desta. Com isso, foram realizadas 
no total oito oficinas (uma teste, seis regionais sendo duas referentes à região sudeste e uma com conselheiros de saúde), entre o período de setembro de 2013 a abril de 2014 .

A realização da oficina teste em setembro de 2013 possibilitou identificar a necessidade de ampliar o processo de consulta para a revisão da PNPS, com a agregação de outras abordagens metodológicas que viabilizassem a consulta a especialistas e outros atores chaves, além da ampliação da escuta sobre os alcances e limites da PNPS, publicada em 2006.

A partir destas reflexões e constatações, um conjunto de estratégias foi aprovado pelo Comitê Gestor da PNPS e organizadas conforme segue:

1) ampliação da escuta de profissionais de saúde, representantes de movimentos sociais, gestores, técnicos de secretarias e departamentos do Ministério da Saúde e interessados em geral, a partir de uma plataforma de questionários eletrônicos vinculados ao domínio da rede mundial de computadores, gerenciada pelo Ministério da Saúde, denominada amplamente como "FormSUS". Este instrumento foi composto por 51 questões, predominantemente, quantitativas. As questões qualitativas apresentadas possibilitaram a expressão da opinião e sugestão do participante com limite de 80 caracteres, sendo estas vinculadas a alguma questão quantitativa. Este foi preenchido por 1545 , respondentes no período de 01 de dezembro de 2013 a 23 de março de 2014, tendo os representantes listados no Quadro 1 recebido o convite formalizado pelo próprio Ministério da Saúde. Adicionalmente o formulário esteve disponibilizado no site do Ministério da Saúde, para que usuários, trabalhadores de saúde, representantes de universidades e de movimentos sociais pudessem também colaborar. A forma como o questionário foi estruturado exigia dos respondentes uma familiaridade com a PNPS o que pode ter resultado em uma maior divulgação da mesma entre os segmentos consultados.
2) Realização de consulta intrassetorial por meio da técnica Delphi de construção de consenso com informantes chaves do Ministério da Saúde e universidades (pesquisadores e professores). A técnica Delphi foi definida por Westphal e Minowa ${ }^{39}$ "como um processo de consulta a distância a um grupo de especialistas que não tem comunicação face-a-face entre si, sobre um determinado assunto", nesse caso a PNPS.

3) Construção de consenso com informantes chaves de outros Ministérios e Secretarias do Governo, bem como de instituições não governamentais selecionadas, caracterizando uma consulta intersetorial, também por meio da utilização do método Delphi.

4) Os produtos dos diferentes movimentos simultâneos foram então trabalhados por meio de quatro encontros de sistematização, coordenados pelo GT Promoção da Saúde da Abrasco, realizados entre março a maio de 2014. Estes se constituíram em um espaço privilegiado de debate, reflexão e construção de eixos comuns que possibilitaram a triangulação dos resultados.

Nestes encontros presenciais de sistematização, para a condução da leitura triangulada dos movimentos, foi construída uma matriz de análise dos diferentes movimentos simultâneos (Quadro 1) buscando sistematizar elementos de coerência e construção de sentidos nos resultados, contemplando elementos como concepções, objetivos, diretrizes, estratégias, temas, princípios e valores.

Esta matriz possibilitou o agrupamento de todos os componentes da PNPS trabalhados durantes os diferentes movimentos e encontrar outros dois que emergiram no processo de triangulação: Estratégias e Temas Transversais, que passaram a compor a proposta de minuta da PNPS revisada (Figura 3).

5) Como última etapa deste processo de revisão do texto da PNPS, foi realizado um seminário nacional em Brasília, em maio de 2014, com

Quadro 1. Matriz de análise dos diferentes movimentos simultâneos, Brasil, 2014.

\begin{tabular}{|c|c|c|c|c|c|c|c|c|}
\hline Movimento: & $\begin{array}{c}\text { Concepções } \\
\text { Conceitos }\end{array}$ & $\begin{array}{c}\text { Princípios } \\
\text { Valores }\end{array}$ & Objetivos & Diretrizes & Temas & $\begin{array}{c}\text { Eixos } \\
\text { Operacionais }\end{array}$ & Estratégias & Recomendações \\
\hline & & & & & & & \\
\hline
\end{tabular}




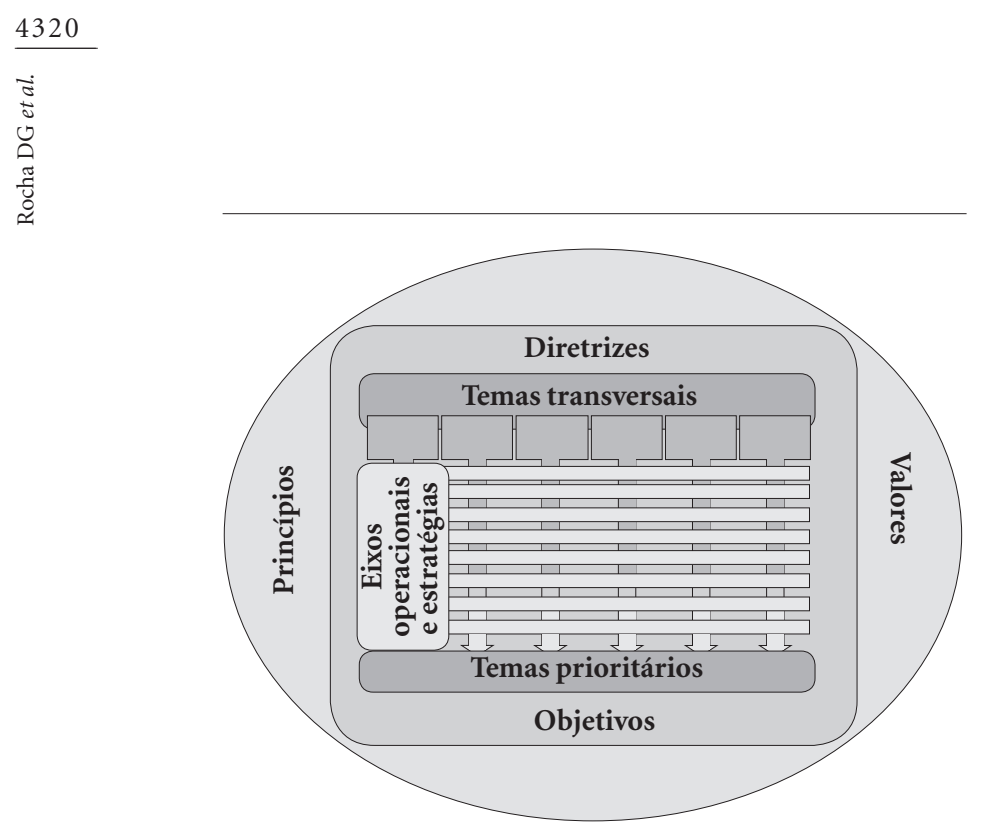

Figura 3. Mariz de conexão entre os componentes da Política Nacional de Promoção da Saúde, Brasil, 2014.

aproximadamente 300 participantes. Este teve como objetivos a apresentação dos resultados das diferentes abordagens metodológicas e da metaleitura, assim como uma síntese do texto revisado. Este momento foi considerado pelo grupo condutor como mais uma etapa de validação por pares tendo em vista que participaram do seminário representantes dos diversos segmentos ouvidos pelos Delphi, oficinas regionais e questionário eletrônico.

Pode-se afirmar que estes diversos procedimentos metodológicos, realizados simultaneamente, culminaram em uma ampla escuta dos atores envolvidos ou com potencial de envolvimento com o campo da promoção da saúde no Brasil.

\section{Considerações finais}

A partir da análise do número de participantes segundo segmento e estratégia de escuta ou mobilização, e considerando os múltiplos movimentos simultâneos, pode-se afirmar que houve ampliação da participação social nesta revisão da PNPS, cumprindo com o objetivo de garantir a representatividade democrática na formulação de políticas e a revisão como um caminho para superar este déficit na formulação de 2002 e 2006.

Entre os ganhos resultantes deste processo, destaca-se que a aproximação das realidades regionais e a problematização das práticas de promoção da saúde implementadas em diferen- tes contextos do território brasileiro, registradas durante a realização das oficinas regionais. Isto possibilitou a identificação das convergências e singularidades da implementação da PNPS vigente e a caracterização da diversidade socioespacial das regiões para subsidiar a definição das estratégias e dos objetivos da nova política. $\mathrm{O}$ reconhecimento da relevância da Atenção Básica e da Estratégia Saúde da Família para a implementação da promoção da saúde no SUS, foi um consenso entre todos os atores.

Ainda que não fosse objetivo avaliar a implementação dos oito anos da PNPS, foi possível identificar os principais fatores críticos, segundo a visão dos executores ou implementadores, para a concretização desta no cotidiano e a partir do SUS.

Constatou-se que o processo de revisão, os encontros de sistematização e a escrita compartilhada da minuta da PNPS revisada favoreceram a construção de consensos e poderão contribuir para a produção de um "glossário", ou pacto semântico, que vai além das terminologias e linguagens de "experts" ou acadêmicos.

Tem-se a expectativa de que a rede de compromissos e interesses mobilizados nas diferentes regiões (oficinas e cartões assinados e enviados para o Comitê Gestor da PNPS) e nos espaços de pactuação do SUS, durante os múltiplos movimentos da revisão tenha sustentabilidade; com a continuidade do advocacy, comunicação e identificação de estratégias para inclusão das diretrizes, dos princípios e valores da Promoção da Saúde nos Planos de Ação setoriais e intersetoriais locorregionais.

Entre os próximos passos referentes aos compromissos e responsabilidades dos atores envolvidos com a nova PNPS observa-se que, até o momento, estes continuam empenhados em aprovar a nova política nas instâncias de pactuação do SUS e realizar o lançamento nacional até outubro de 2014. Estes seguem orientados pela visão de que uma política pública nacional pode ser protagonizada pelo gestor federal, mas, sem que este "engesse a agenda". É muito importante disseminar as diretrizes e formas de financiamento considerando os contextos locais.

A forma como os questionários do FormSUS e DELPHI foi estruturada exigia dos respondentes uma familiaridade com a PNPS, o que pode ter resultado em uma maior divulgação da mesma entre os segmentos consultados.

As agendas internacionais de "Saúde em Todas as Políticas" e das ações da "Declaração Política do Rio sobre os Determinantes Sociais da Saú- 
de" continuam demandando discussões e eventos que resgatem a pertinência dos eixos temáticos e estratégicos inseridos na PNPS revisada.

Esta publicação e outras oriundas deste processo de revisão da política podem contribuir para a produção e difusão do conhecimento na área e subsidiar diretrizes e mecanismo de pesquisa, formação e educação permanente na temática.

A parceria estabelecida entre representantes de uma instituição não governamental (GT Promoção da Saúde da Abrasco) e equipe governamental (representantes do Ministério da Saúde) mediada pela OPAS, visando a ampliação da participação e capilaridade regional do processo de formulação de uma política, sinaliza novos arranjos de advocacy e boa governança mediante a criação de espaços de articulação intergovernamental e intersetorial. Alguns registros das oficinas regionais e encontros sistematização evidenciam que o aprendizado interregional, intersetorial e intergeracional propiciado pelo trabalho coletivo e pela validação de cada um dos movimentos pelos pares e parceiros foi, também, um legado importante do processo.

Finaliza-se ressaltando as contribuiçõoes deste processo para o fortalecimento da agenda estratégica da Saúde Coletiva no Brasil. A promoção da saúde comprometida com o enfrentamento das inequidades intra e interregionais tem potência para fortalecer os princípios doutrinários e organizativos do SUS e favorecer a promoção da equidade.

\section{Colaboradores}

DG Rocha e VC Marcelo trabalharam na concepção, revisão de literatura, escrita e aprovação da versão final; VP Alexandre, R Rezende e R Franco de Sá contribuíram na análise documental, organização dos resultados, escrita e formatação; JD Nogueira na escrita e formatação final do artigo.

\section{Agradecimentos}

Os autores deste artigo não poderiam deixar de reconhecer as outras pessoas que foram ativadores fundamentais dos múltiplos movimentos simultâneos que redundou na Revisão da PNPS: Simone Moyses, Marcia Westphal, Izabelle Vianna, Biatriz Cardoso, Wanessa Almeida, Kleber Rangel Silva, Kenia Lara, Ana Germani, Monica Andrade Morraye, Ana Sperandio, Roberta Amorim, Geórgia Maria de Albuquerque, Fabio Fortunato Brasil de Carvalho, Solena Kusma, Roberto Eduardo Bueno, Jacqueline Lima, Socorro Freire, Maria Socorro Araújo Dias, Maria de Fatima Lobato Tavares, Rogéria Nunes, Kátia Edmundo, Antônio José Cardoso, Deborah Malta Marta Maria Alves da Silva, Patrícia Constant Jaime, Marco Akerman.

O processo de revisão da PNPS contou com recursos do Termo de Cooperação Técnica 81, celebrado entre o Ministério da Saúde e a Organização Pan-americana de Saúde/OMS.

\section{Referências}

1. Campos GW, Barros RB, Castro AM. Avaliação de Política Nacional de Promoção da Saúde. Cien Saude Colet 2004; 9(3):745-749.

2. Traverso-Yèpez MA. Dilemas na promoção da saúde no Brasil: reflexões em torno da política nacional. Interface (Botucatu) 2007; 11(22):223-238.

3. Buss PM, Carvalho AI. Desenvolvimento da promoção da saúde no Brasil nos últimos vinte anos (1988-2008). Cien Saude Colet 2009; 14(6):2305-2316.

4. Rocha DG. O movimento da promoção da saúde na década de 1990: um estudo do seu desenvolvimento e difusão na saúde pública brasileira [tese]. São Paulo: Faculdade de Saúde Pública; 2001.

5. Cruz DKA. Da promoção à prevenção: o processo de formulação da Política Nacional de Promoção da Saúde no período de 2003-2006 [dissertação]. Recife: Centro de Pesquisas Aggeu Magalhães; 2010.

6. Brasil. Conselho Nacional de Secretários de Saúde (CONASS). Atenção primária e promoção da saúde. Brasília: CONASS; 2011.

7. Sousa MF, Hamman EM. Programa Saúde da Família no Brasil: uma agenda incompleta? Cien Saude Colet 2009; 14(Sup. 1):1325-1335.

8. Brasil. Ministério da Saúde (MS). Secretaria de Vigilância em Saúde. Política Nacional de Promoção da Saúde. Brasília: MS; 2006. 
9. Rocha DG, Akerman M. Determinação social da saúde e promoção da saúde: isto faz algum sentido para a Estratégia de Saúde da Família? Em que sentido podemos seguir? In: Sousa MF, Franco MS, Mendonça AVM, organizadores. Saúde da Família nos municípios brasileiros: Os reflexos dos 20 anos no espelho do futuro. Campinas, São Paulo: Saberes; 2014. p. 720-754.

10. Machado CV, Baptista TWF, Lima LD. O planejamento nacional da política de saúde no Brasil: estratégias e instrumentos nos anos 2000. Cien Saude Colet 2010; 15(5):2367-2382.

11. Loureiro I, Miranda N. Promover a saúde. Dos fundamentos à acção. Coimbra: Almedina; 2010.

12. Salinas JC, Vio Del RF. Promoción de la salud en Chile. Rev Chil Nutr 2002; 29(Supl. 1):164-173.

13. Souza C. Políticas públicas: questões temáticas e de pesquisa. Caderno CRH 2003; 39(16):11-24.

14. Brasil. Ministério da Saúde (MS). Política Nacional de Promoção da Saúde (versão preliminar). 2002. [acessado 2014 jul 2]. Disponível em: http://bvsms.saude.gov.br/ bvs/publicacoes/ politica_nac_prom_saude.pdf

15. Pinder $\mathrm{L}$. The federal role in health promotion: under the radar. In: O’Neill M, Pederson A, Dupéré S, Rootman I. Health promotion in Canada. Critical perspectives. $2^{\text {nd }}$. Toronto: Canadian Scholars' Press; 2007. p. 92-105.

16. Rocha DG, Zancan L, Franco De Sá R, Akerman M. 10 anos do Grupo Temático Promoção da Saúde e Desenvolvimento Local-ABRASCO. [acessado 2013 nov 15]. Disponível em: http://www.abrasco.org.br/site/sites/gt promocaodasaude

17. Zancan L, Rocha DG, Franco De Sá R. Grupo Temático Promoção da Saúde. Relatório Final de Oficina Pré- IX Congresso Brasileiro de Saúde Coletiva Recife -Pernambuco; 2009. [acessado 2013 out 02]. Disponível em: http://www.abrasco.org.br/site/sites/gtpromocaoda saude

18. Grupo Temático de Promoção da Saúde, Abrasco. Comitê Gestor da Política Nacional de Promoção da Saúde do Ministério da Saúde. Pontos para o diálogo sobre "Saúde em Todas as Políticas" (STP): em busca de uma posição brasileira. Brasília: GT PS DLIS-MS; 2013.

19. Lima LD, Viana ALA, Machado CV, Albuquerque MV, Oliveira RG, Iozzi FL, Scatena JHG, Mello GA, Pereira AMM, Coelho APS. Regionalização e acesso à saúde nos estados brasileiros: condicionantes históricos e político-institucionais. Cien Saude Colet 2012; 17(11):2881-2892.20.

20. Cortes SV. Sistema Único de Saúde: espaços decisórios e a arena política de saúde. Cad Saude Publica 2009; 25(7):1626-1633.

21. Coelho JS. Construindo a participação social no SUS: um constante repensar em busca de equidade e transformação. Saúde Soc 2012; 21(Supl. 1):138-151.

22. Shimizu HE, Pereira MF, Cardoso AJC, Bermudez XPCD. Representações sociais dos conselheiros municipais acerca do controle social em saúde no SUS. Cien Saude Colet 2013; 18(8):2275-2284.

23. Araujo Júnior LC, Maciel Filho R. Developing an operational framework for health policy analysis. Revista Brasileira de Saúde Materno Infantil 2001; 1(3):203221.

24. Buss P, Pellegrini A. A Saúde e seus determinantes sociais. Physis: Rev. Saúde Coletiva 2007; 17(1):77-93.
25. Marmot M, Allen J. Prioritizing health equity. In: Leppo K, Ollila E, Peña S, Wismar M, Cook S, editores. Health in all policies. Seizing opportunities, implementing policies. Finland: Ministry of Social Affairs and Health; 2013. p. 63-80.

26. Brasil. Ministério da Saúde (MS). Secretaria de Vigilância à Saúde. Departamento de Análise de Situação de Saúde. Plano de ações estratégicas para o enfrentamento das doenças crônicas não transmissíveis (DCNT) no Brasil. 2011-2022. Brasília: MS; 2011.

27. Brasil Presidência da República. Casa Civil. Subchefia para Assuntos Jurídicos. Decreto no 7.508, de 28 de junho de 2011. Diário Oficial da União 2011; 29 jun.

28. World Health Organization (WHO). Global Forum. Addressing the challenge of noncommunicable diseases. Forum Report; 2011. [acessado 2014 jul 15]. Disponível em: http://www.who.int/nmh/events/global_forum _ncd/en/.

29. World Health Organization (WHO). Rio Political Declaration on Social Determinants of Health. Rio de Janeiro: WHO; 2011.

30. Organização das Nações Unidas (ONU). Declaração do Rio sobre Meio Ambiente e Desenvolvimento. Rio de Janeiro: ONU; 2012.

31. World Health Organization (WHO). The Helsinki Statement on Health in All Policies. The 8th Global Conference on Health Promotion. Helsinki-Finland; 2013. [acessado 2014 jun 10]. Disponível em: http://health promotion2013.org/images/8GCHP_Helsinki_State ment.pdf

32. Brasil. Ministério da Saúde (MS). Secretaria de Vigilância à Saúde. Secretaria da Atenção à Saúde. Política Nacional de Promoção da Saúde. $3^{\mathrm{a}}$ ed. Brasília: MS; 2010.

33. Serafim MP, Dias RB. Análise de Políticas Públicas: uma revisão da literatura. Cad Gestão Social 2012; 3(1):121-134.

34. Minayo MCS. Análise qualitativa: teoria, passos e fidedignidade. Cien Saude Colet 2012; 17(3):621-626.

35. Rocha DG, Rezende R, Moysés ST, Franco de Sá R. Metodologia das oficinas regionais de revisão da Política $\mathrm{Na}$ cional de Promoção da Saúde. Brasília: Abrasco; 2013.

36. Organização Pan-Americana de Saúde (OPAS). Guia de Avaliação Participativa de Municípios e Comunidades Saudáveis: recursos práticos para a ação. São Paulo: OPAS; 2005.

37. Fetterman DM. Empowerment evaluation. Evaluation Practice 1994; 15(1):1-15.

38. King C, Rootman I, Dupéré S, Pederson A, O’Neill M. Final report of the Pre Conference Workshop at the Canadian Public Health Conference on the future of health promotion in Canada. Victoria: Public Health Association of British Columbia; 2012.

39. Westphal MF, Minowa E. Documento técnico com síntese dos resultados da consulta intrassetorial e com as IES/universidades sobre o eixo II do Plano Nacional de Enfrentamento das Doenças Crônicas a ser apresentado nas Oficinas de Consenso previstas pela metodologia de Revisão da Política Nacional de Promoção da Saúde. Brasília: Ministério da Saúde (MS), OPAS; 2014.

Artigo apresentado em 05/08/2014

Aprovado em 15/08/2014

Versão final apresentada em 16/08/2014 